Article

\title{
On Analytical Solutions of the Fractional Differential Equation with Uncertainty: Application to the Basset Problem
}

\author{
Soheil Salahshour ${ }^{1, *}$, Ali Ahmadian ${ }^{2}$, Norazak Senu ${ }^{2}$, Dumitru Baleanu ${ }^{3,4}$ and \\ Praveen Agarwal ${ }^{5}$ \\ ${ }^{1}$ Young Researchers and Elite Club, Mobarakeh Branch, Islamic Azad University, Mobarakeh, Iran \\ ${ }^{2}$ Department of Mathematics, Faculty of Science, Universiti Putra Malaysia, 43400UPM, Serdang, \\ Selangor, Malaysia; E-Mails: ahmadian.hosseini@gmail.com (A.A.); norazak@upm.edu.my (N.S.) \\ ${ }^{3}$ Department of Mathematics and Computer Sciences, Faculty of Art and Sciences, \\ Cankaya University, Balgat 0630, Ankara, Turkey; E-Mail: dumitru@ cankaya.edu.tr \\ ${ }^{4}$ Institute of Space Sciences, P.O.Box MG-23, Magurele-Bucharest, Romania \\ ${ }^{5}$ Department of Mathematics, Anand International College of Engineering, Jaipur-303012, India; \\ E-Mail: goyal.praveen2011@gmail.com
}

* Author to whom correspondence should be addressed; E-Mail: soheilsalahshour@yahoo.com; Tel.:+98-915-5154014.

Academic Editor: Carlo Cafaro

Received: 24 November 2014 / Accepted: 15 January 2015 / Published: 16 February 2015

\begin{abstract}
In this paper, we apply the concept of Caputo's H-differentiability, constructed based on the generalized Hukuhara difference, to solve the fuzzy fractional differential equation (FFDE) with uncertainty. This is in contrast to conventional solutions that either require a quantity of fractional derivatives of unknown solution at the initial point (Riemann-Liouville) or a solution with increasing length of their support (Hukuhara difference). Then, in order to solve the FFDE analytically, we introduce the fuzzy Laplace transform of the Caputo H-derivative. To the best of our knowledge, there is limited research devoted to the analytical methods to solve the FFDE under the fuzzy Caputo fractional differentiability. An analytical solution is presented to confirm the capability of the proposed method.
\end{abstract}

Keywords: fuzzy fractional differential equation; fuzzy Laplace transform; Caputo differentiability; dynamical systems; Basset problem 


\section{Introduction}

The fractional differential equation (FDE) is gaining more attention, since it is capable of modeling different processes in physics, chemistry and engineering (see [1-7]). Furthermore, it appeared in the theory of the control of dynamical systems, when the controlled system and/or the controller is described by an FDE [8-10]. Some relevant monographs are Podlubny [10], Kilbas et al. [11] and Baleanu et al. [12]. Accordingly, providing the analytical and numerical methods to solve the FDEs has become an active research undertaking (see [12-22]). Furthermore, one reason for the interest in FDE comes from applications that involve new ways of modeling physical systems using tools from fractional calculus. For example, consider the dynamics of a system that involves the motion of a rheological specimen that exhibits both elasticity and dissipation [9,23]. An alternative approach instead of the traditional models would be to combine the effects of stiffness and damping in a single term by an FDE:

$$
\begin{aligned}
D_{a}^{\beta} x(t) & =f(t, x(t)), 0<\beta \leq 1, t>a, \\
x\left(t_{0}\right) & =x_{0},
\end{aligned}
$$

where we usually could not be sure that the model is true. One of the main reasons is that the initial value in Equation (1) may not be known precisely, as it is usually characterized by a domain expert. Therefore, instead of using the deterministic values, one of the other popular choices is to employ the fuzzy initial value. That is, each element of the FDE is converted to the fuzzy frame, and this is how the fuzzy fractional differential equation (FFDE) emerged.

Moreover, deducing equations of dynamics from empirical observations is fundamental in science. Furthermore, describing and understanding natural phenomena is the goal of theoretical physics and mathematics. Theoretical physics has had a general measure of the uncertainty associated with the behavior of a probabilistic process for more than 100 years [24,25]. Over the past two decades, a new mathematical description has been formulated, linked together by tools of fractional calculus, which include fractional constitutive laws, probabilistic models based on continuous time random walks and generalized central limit theorems, fractional Langevin equations, fractional Brownian motions, fractional diffusion and the fractional Fokker-Planck equation. Commonly, the unknown parameters involved in such models are assumed constant over time. In reality, however, some of them are not constant and implicitly depend on several factors. Many such factors usually do not appear explicitly in the mathematical models, due to the tradeoffs between modeling and numerical tractability and the lack of precise knowledge about them. In order to deal with such uncertainty in those parameters, a stochastic approach is commonly employed with the assumption that stochastic behavior implies knowledge of the probabilistic information of the system components. However, this information can be very complicated, with errors and vagueness. With this motivation, the authors provide a different approach to depict such physical models with the parameters and initial values defined under fuzzy fractional setting theory. This interpretation can be closer to the origin of the physical models, and it has enough ability to be extended to the other models in addition to the currently discussed one.

One of the pioneering works in fuzzy fractional calculus was conducted by Agarwal et al. in [26], where they proposed a concept for the FDE with uncertainty. In this paper, the Riemann-Liouville differentiability equipped with a fuzzy initial condition to solve the FFDE was used. We recall that the differentiability of the fuzzy-valued functions in the fractional case was not covered. To extend 
this, Allahviranloo et al. [27] introduced the Riemann-Liouville H-differentiability in order to solve the FFDEs, which is a direct generalization of the fractional Riemann-Liouville derivative using the Hukuhara difference. Afterward, Mazandarani and Vahidian Kamyad [28] introduced the Caputo-type fuzzy fractional derivative for the solution of FFDE. Recently, Salahshour et al. [29] used the fuzzy Laplace transform [30] in order to solve analytically such problems, which was followed up by Mazandarani and Najariyan [31] to introduce the fuzzy Laplace transform under Type 2 fuzzy fractional differentiability. However, there are three flaws associated with these aforementioned solutions. In the former, as highlighted in [32,33], there is a limitation in the Hukuhara difference, where it leads to solutions with increasing length of their support. In the latter, the Riemann-Liouville derivative requires a quantity of the fractional derivative of unknown solution at the initial point, and in the last one, the authors applied the method under Type 2 fuzzy setting theory, which increases the computational cost, although it is closer to the originality of the model. Practically, we do not clearly know what is the meaning of the fractional derivative at that point. In other words, the required quantity could not be measured and perhaps may not exist. Due to this important drawback, a few papers were published to consider Caputo's fuzzy differentiability, as well as numerical methods for the solution of FFDEs [28,34-36], which is still traceless and unknown.

In this manuscript, we propose a different mathematical model based on the FFDE and present its analytical solution. Particularly, we employ the strongly-generalized differentiability based on the Caputo derivative, as in [28,37]. As a result, the proposed solution will allow us to handle the limitations suffered by the Hukuhara difference and Riemann-Liouville differentiability, respectively. Then, we employ the fuzzy Laplace transforms method [29,38] to solve the FFDEs analytically. This is in contrast to the solution in [37], where they derived an equivalent integral form of FFDE [39] to obtain the approximate solutions. We refer to some works on the fuzzy Laplace transforms in [30,40,41] and [29] and the references therein. Recently, applying the fuzzy Laplace transform has been widely used in order to solve fuzzy fractional differential equations; see [31,38]. In fact, for the first time in the literature, we have solved a mixed type of fractional differential equation under uncertainty (including natural and fractional order), the so-called fuzzy fractional Basset equation.

The paper is organized as follows: We revisit briefly some of the well-known definitions of the fuzzy numbers in Section 2 and Caputo's H-differentiability in Section 3, respectively. The fuzzy Laplace transforms are used for the fuzzy-valued function and the novel theorem for the Laplace transform of $f$ under Caputo's H-derivative detailed in Section 4. The solutions of the FFDE using the fuzzy Laplace transforms and their inverses are investigated in Section 5. In Section 6, some real examples in the fuzzy sense are solved to show the presented method. Finally, the conclusion is drawn in Section 7.

\section{Preliminaries}

We recall that the basic definition of the fuzzy numbers had been discussed (see [42,43]), and interested readers are encouraged to read them for the details. We denote the set of all real numbers as $R$ and the set of all fuzzy numbers on $R$ as $E$.

Therefore, a fuzzy number is a mapping $u: R \rightarrow[0,1]$ that satisfies the following properties:

(1) $u$ is upper semi-continuous, 
(2) $u$ is fuzzy convex, i.e., for all $x, y \in R, \lambda \in[0,1]: u(\lambda x+(1-\lambda) y) \geq \min \{u(x), u(y)\}$,

(3) $u$ is normal, i.e., $\exists x_{0} \in R$ for which $u\left(x_{0}\right)=1$,

(4) $\operatorname{supp} u=\{x \in R \mid u(x)>0\}$ is the support of the $u$, and its closure $c l$ (supp $u$ ) is compact.

Consequently, a similar definition, but in parametric form, is provided in [44] as:

Definition 1. A fuzzy number $u$ in parametric form is a pair $(\underline{u}, \bar{u})$ of functions $\underline{u}(r), \bar{u}(r), 0 \leq r \leq 1$, where the following requirements hold:

(1) $\underline{u}(r)$ is a bounded non-decreasing left continuous function in $(0,1]$ and right continuous at zero,

(2) $\bar{u}(r)$ is a bounded non-increasing left continuous function in $(0,1]$ and right continuous at zero,

(3) $\underline{u}(r) \leq \bar{u}(r), 0 \leq r \leq 1$.

Definition 2. Let $E$ be the set of all fuzzy numbers on $R$. The r-level set of a fuzzy number $u \in E$, $0 \leq r \leq 1$, denoted by $[u]^{r}$, is defined as:

$$
[u]^{r}= \begin{cases}\{x \in R \mid u(x) \geq r\}, & \text { if } 0<r \leq 1 \\ \operatorname{cl}(\operatorname{supp} u), & \text { if } r=0\end{cases}
$$

It is clear that the r-level set of a fuzzy number is a closed and bounded interval $[\underline{u}(r), \bar{u}(r)]$, where $\underline{u}(r)$ denotes the left-hand endpoint of $[u]^{r}$ and $\bar{u}(r)$ denotes the right-hand endpoint of $[u]^{r}$.

Regarding Zadeh's extension principle, the "addition" operation $(\oplus)$ on $E$ is presented as:

$$
(u \oplus v)(x)=\sup _{y \in R} \min \{u(y), v(x-y)\}, \quad x \in R,
$$

and the scalar multiplication of a fuzzy number is described as:

$$
(k \odot u)(x)=\left\{\begin{array}{cc}
u(x / k), & k>0 \\
\widetilde{0}, & k=0
\end{array}\right.
$$

where $\tilde{0} \in E$. It is well known that these properties are true for all levels of:

$$
[u \oplus v]^{r}=[u]^{r}+[v]^{r}, \quad[k \odot u]^{r}=k[u]^{r} .
$$

The Hausdorff distance between these fuzzy numbers is indicated by $d: E \times E \longrightarrow[0, \infty]$,

$$
d(u, v)=\sup _{r \in[0,1]} \max \{|\underline{u}(r)-\underline{v}(r)|,|\bar{u}(r)-\bar{v}(r)|\},
$$

where $u(r)=[\underline{u}(r), \bar{u}(r)], v(r)=[\underline{v}(r), \bar{v}(r)]$ is utilized in [32,44]. From here, one can easily find that $d$ is a metric in $E$ and has the following properties [45]:

(a) $d(u \oplus w, v \oplus w)=d(u, v), \quad \forall u, v, w \in E$,

(b) $d(k \odot u, k \odot v)=|k| d(u, v), \quad \forall k \in R, u, v \in E$, 
(c) $d(u \oplus v, w \oplus e) \leq d(u, w)+d(v, e), \quad \forall u, v, w, e \in E$,

(d) $(E, d)$ is a complete metric space.

Definition 3. A mapping $f: R \times E \rightarrow E$ is continuous at point $\left(x_{0}, y_{0}\right) \in R \times E$, provided that for any fixed $r \in[0,1]$ and arbitrary $\epsilon>0$, there exists an $\delta(\epsilon, r)$, such that:

$$
d\left([f(x, y)]^{r},\left[f\left(x_{0}, y_{0}\right)\right]^{r}\right)<\epsilon,
$$

whenever $\left|x-x_{0}\right|<\delta(\epsilon, r)$ and $d\left([y(x)]^{r},\left[y_{0}\right]^{r}\right)<\delta(\epsilon, r)$ for all $x \in R$ and $y \in E$.

Theorem 1. (see [46]) Let $f(x)$ be a fuzzy-valued function on $[a, \infty)$, and it is represented by $(\underline{f}(x ; r), \bar{f}(x ; r))$. For any fixed $r \in[0,1]$, assume $\underline{f}(x ; r)$ and $\bar{f}(x ; r)$ are Riemann-integrable on $[a, b]$ for every $b \geq a$, and assume that there are two positive functions $\underline{M}(r)$ and $\bar{M}(r)$, such that $\int_{a}^{b}|\underline{f}(x ; r)| d x \leq \underline{M}(r)$ and $\int_{a}^{b}|\bar{f}(x ; r)| d x \leq \bar{M}(r)$ for every $b \geq a$; then, $f(x)$ is improper fuzzy Riemann-integrable on $[a, \infty)$. The improper fuzzy Riemann-integral is a fuzzy number, and we have:

$$
\int_{a}^{\infty} f(x ; r) d x=\left[\int_{a}^{\infty} \underline{f}(x ; r) d x, \int_{a}^{\infty} \bar{f}(x ; r) d x\right]
$$

Definition 4. Let $x, y \in E$, such that $E$ is the set of all fuzzy numbers on $R$; if there exists $z \in E$, such that $x=y \oplus z$, then $z$ is called the H-difference of $x$ and $y$ and is denoted as $x \ominus y$. In this paper, the sign “ $\ominus$ ” always refers to the $H$-difference, unless specified otherwise, and also, note that $x \ominus y \neq x+(-1) y$.

Definition 5. Let $f:(a, b) \rightarrow E$ and $x_{0} \in(a, b)$. $f$ be called strongly-generalized differentiable at $x_{0}$, if an element $f^{\prime}\left(x_{0}\right) \in E$ exists, such that:

(1) for all $h>0$ sufficiently small, $\exists f\left(x_{0}+h\right) \ominus f\left(x_{0}\right), \exists f\left(x_{0}\right) \ominus f\left(x_{0}-h\right)$ and the limits (in the metric d): $\lim _{h \searrow 0} \frac{f\left(x_{0}+h\right) \ominus f\left(x_{0}\right)}{h}=\lim _{h \searrow 0} \frac{f\left(x_{0}\right) \ominus f\left(x_{0}-h\right)}{h}=f^{\prime}\left(x_{0}\right)$,

or:

(2) for all $h>0$ sufficiently small, $\exists f\left(x_{0}\right) \ominus f\left(x_{0}+h\right), \exists f\left(x_{0}-h\right) \ominus f\left(x_{0}\right)$ and the limits (in the metric d): $\lim _{h \searrow 0} \frac{f\left(x_{0}\right) \ominus f\left(x_{0}+h\right)}{-h}=\lim _{h \searrow 0} \frac{f\left(x_{0}-h\right) \ominus f\left(x_{0}\right)}{-h}=f^{\prime}\left(x_{0}\right)$,

or:

(3) for all $h>0$ sufficiently small, $\exists f\left(x_{0}+h\right) \ominus f\left(x_{0}\right), \exists f\left(x_{0}-h\right) \ominus f\left(x_{0}\right)$ and the limits (in the metric d): $\lim _{h \searrow 0} \frac{f\left(x_{0}+h\right) \ominus f\left(x_{0}\right)}{h}=\lim _{h \searrow 0} \frac{f\left(x_{0}-h\right) \ominus f\left(x_{0}\right)}{-h}=f^{\prime}\left(x_{0}\right)$,

or:

(4) for all $h>0$ sufficiently small, $\exists f\left(x_{0}\right) \ominus f\left(x_{0}+h\right), \exists f\left(x_{0}\right) \ominus f\left(x_{0}-h\right)$ and the limits (in the metric d): $\lim _{h \searrow 0} \frac{f\left(x_{0}\right) \ominus f\left(x_{0}+h\right)}{-h}=\lim _{h \searrow 0} \frac{f\left(x_{0}\right) \ominus f\left(x_{0}-h\right)}{h}=f^{\prime}\left(x_{0}\right)$ ( $h$ and $-h$ at denominators mean $\frac{1}{h}$ and $\frac{-1}{h}$, respectively).

It is important to note that, for the sake of simplicity, the function that satisfies Case (1) (or Case (2)) in Definition 5 is known as the (1)-differentiable function (or (2)-differentiable function). The principal properties of these derivatives are well described (see, for example, [32,33,47]), and the following theorems are adopted. 
Theorem 2. (see [47]) Let $f: R \rightarrow E$ be a function, and denote $f(x ; r)=[\underline{f}(x ; r), \bar{f}(x ; r)]$, for each $r \in[0,1]$.

(1) If $f$ is a (1)-differentiable function, then $\underline{f}(x ; r)$ and $\bar{f}(x ; r)$ are differentiable functions and $\left[f^{\prime}(x)\right]^{r}=\left[\underline{f}^{\prime}(x ; r), \bar{f}^{\prime}(x ; r)\right]$

or:

(2) If $f$ is a (2)-differentiable function, then $\underline{f}(x ; r)$ and $\bar{f}(x ; r)$ are differentiable functions and $\left[f^{\prime}(x)\right]^{r}=\left[\bar{f}^{\prime}(x ; r), \underline{f}^{\prime}(x ; r)\right]$.

\section{Caputo's H-Differentiability}

In this section, the concept of the fuzzy Caputo derivative as in [29] is revisited using the Hukuhara difference, and interested readers are encouraged to see the full details in the aforementioned paper. We indicate $C(J, E)$ as a space of all fuzzy-valued functions, continuous on $J=(a, b]$ [37]:

$$
C(J, E):=\{f \mid f:(a, b] \longrightarrow E, f \text { is continuous }\} .
$$

where, throughout the paper, we hypothesized that the fuzzy-valued function $f$ is located in $C(J, E)$, unless specified otherwise.

Definition 6. Let $f:[a, b] \longrightarrow E$, for $0<\beta \leq 1$; the fuzzy Riemann-Liouville integral of fuzzy-valued function $f$ is defined as:

$$
\left(I_{a+}^{\beta} f\right)(x)=\frac{1}{\Gamma(\beta)} \int_{a}^{x} \frac{f(t) d t}{(x-t)^{1-\beta}}, x>a .
$$

For all $0 \leq r \leq 1$, since $f(x ; r)=[\underline{f}(x ; r), \bar{f}(x ; r)]$, the fuzzy Riemann-Liouville integral of fuzzy-valued function $f$ can be calculated based on its lower function and upper function, respectively, as follows:

Theorem 3. (see [37]) Let $f:[a, b] \longrightarrow E$, for $0 \leq r \leq 1$ and $0<\beta \leq 1$; the fuzzy Riemann-Liouville integral of fuzzy-valued function $f$ is defined as:

$$
\left(I_{a+}^{\beta} f\right)(x ; r)=\left[\left(I_{a+}^{\beta} \underline{f}\right)(x ; r),\left(I_{a+}^{\beta} \bar{f}\right)(x ; r)\right],
$$

where

$$
\left(I_{a+\underline{f}}^{\beta}\right)(x ; r)=\frac{1}{\Gamma(\beta)} \int_{a}^{x} \frac{\underline{f}(t ; r) d t}{(x-t)^{1-\beta}}, \quad\left(I_{a+}^{\beta} \bar{f}\right)(x ; r)=\frac{1}{\Gamma(\beta)} \int_{a}^{x} \frac{\bar{f}(t ; r) d t}{(x-t)^{1-\beta}} .
$$

Then, the fuzzy Riemann-Liouville fractional derivatives of order $0<\beta<1$ for fuzzy-valued function $f$ (which is a direct extension of strongly-generalized H-differentiability in [32] and the lateral type of H-differentiability in [47] in the fractional literature) are defined as follows:

Definition 7. Let $f:(a, b) \longrightarrow E, x_{0} \in(a, b)$ and $\Phi(x)=\frac{1}{\Gamma(1-\beta)} \int_{a}^{x} \frac{f(t) d t}{(x-t)^{\beta}}$. For all $0 \leq r \leq 1, h>0$. $f(x)$ is called fuzzy Riemann-Liouville fractional differentiable of order $0<\beta<1$ at $x_{0}$, if there exists an element $\left({ }^{R L} D_{a^{+}}^{\beta} f\right)\left(x_{0}\right) \in E$, such that: 
(1)

$$
\left({ }^{R L} D_{a^{+}}^{\beta} f\right)\left(x_{0}\right)=\lim _{h \longrightarrow 0} \frac{\Phi\left(x_{0}+h\right) \ominus \Phi\left(x_{0}\right)}{h}=\lim _{h \longrightarrow 0} \frac{\Phi\left(x_{0}\right) \ominus \Phi\left(x_{0}-h\right)}{h},
$$

or:

(2)

$$
\left({ }^{R L} D_{a^{+}}^{\beta} f\right)\left(x_{0}\right)=\lim _{h \rightarrow 0} \frac{\Phi\left(x_{0}\right) \ominus \Phi\left(x_{0}+h\right)}{-h}=\lim _{h \rightarrow 0} \frac{\Phi\left(x_{0}-h\right) \ominus \Phi\left(x_{0}\right)}{-h} .
$$

For the sake of simplicity, a fuzzy-valued function $f$ is ${ }^{R L}[(1)-\beta]$-differentiable if it is differentiable, as in Definition 7, Case (1), and is $R L[(2)-\beta]$-differentiable if it is differentiable as in Definition 7 , Case (2).

Theorem 4. (see [37]) Let $f:(a, b) \longrightarrow E$ and $x_{0} \in(a, b), 0<\beta<1$; then, for all $0 \leq r \leq 1$, we have the following relations:

(1) If $f$ is $a^{R L}[(1)-\beta]$-differentiable fuzzy-valued function, then:

$$
\left({ }^{R L} D_{a^{+}}^{\beta} f\right)\left(x_{0} ; r\right)=\left[\left({ }^{R L} D_{a^{+}}^{\beta} \underline{f}\right)\left(x_{0} ; r\right),\left({ }^{R L} D_{a^{+}}^{\beta} \bar{f}\right)\left(x_{0} ; r\right)\right]
$$

or:

(2) If $f(x)$ is $a^{R L}[(2)-\beta]$-differentiable fuzzy-valued function, then:

$$
\left({ }^{R L} D_{a^{+}}^{\beta} f\right)\left(x_{0} ; r\right)=\left[\left({ }^{R L} D_{a^{+}}^{\beta} \bar{f}\right)\left(x_{0} ; r\right),\left({ }^{R L} D_{a^{+}}^{\beta} \underline{f}\right)\left(x_{0} ; r\right)\right]
$$

where

$$
\begin{aligned}
\left({ }^{R L} D_{a^{+}}^{\beta} \underline{f}\right)\left(x_{0} ; r\right) & =\left[\frac{1}{\Gamma(1-\beta)} \frac{d}{d x} \int_{a}^{x} \frac{f(t ; r) d t}{(x-t)^{\beta}}\right]_{x=x_{0}} \\
\left({ }^{R L} D_{a^{+}}^{\beta} \bar{f}\right)\left(x_{0} ; r\right) & =\left[\frac{1}{\Gamma(1-\beta)} \frac{d}{d x} \int_{a}^{x} \frac{\bar{f}(t, r) d t}{(x-t)^{1-\beta}}\right]_{x=x_{0}} .
\end{aligned}
$$

This leads to the derivation of the fuzzy Caputo fractional derivatives. Let $[a, b]$ be a finite interval of the real line $R$ and ${ }^{R L} D_{a^{+}}^{\beta}$ be the Riemann-Liouville H-derivative of order $\beta$ as in Definition 7.

Proposition 1. (see [37,48]) Let $b>0$ and $J=(a, b]$; we denote $C(J, E)$ as the space of all continuous fuzzy functions defined on $J$. Furthermore, let $f \in C(J, E)$; we say that $f \in L^{1}(J, E)$ iff $d\left(\int_{a}^{b} f(s) d s, \tilde{0}\right)<\infty$, where $d$ is the fuzzy metric defined in Section 2.

For more details, please see [37,48-50]. The fuzzy Caputo fractional derivatives under the Hukuhara difference $\left({ }^{C} D_{a^{+}}^{\beta} f\right)(x)$ are defined as:

Definition 8. (see [28,37]) Let $f \in C(J, E) \cap L^{1}(J, E)$ be a fuzzy set-value function; then $f$ is Caputo fuzzy $H$-differentiable at $x$ when:

$$
\left({ }^{C} D_{a^{+}}^{\beta} f\right)(x)=\frac{1}{\Gamma(1-\beta)} \int_{a}^{x} \frac{f^{\prime}(t)}{(x-t)^{\beta}} d t
$$

where $0<\alpha<1$; then, we say $f$ is ${ }^{C}[(1)-\beta]$-differentiable if Equation (7) holds, while $f$ is (1)-differentiable, and $f$ is ${ }^{C}[(2)-\beta]$-differentiable if Equation (7) holds, while $f$ is (2)-differentiable. 
Theorem 5. (see [37]) Let $0<\beta<1$ and $f \in C(J, E)$; then the fuzzy Caputo fractional derivative exists on $(a, b)$, and for all $0 \leq r \leq 1$, we have:

$$
\left({ }^{C} D_{a^{+}}^{\beta} f\right)(x ; r)=\left[\frac{1}{\Gamma(1-\beta)} \int_{a}^{x} \frac{f^{\prime}(t)}{(x-t)^{\beta}} d t, \frac{1}{\Gamma(1-\beta)} \int_{a}^{x} \frac{\bar{f}^{\prime}(t)}{(x-t)^{\beta}} d t\right]
$$

when $f$ is (1)-differentiable, and:

$$
\left({ }^{C} D_{a^{+}}^{\beta} f\right)(x ; r)=\left[\frac{1}{\Gamma(1-\beta)} \int_{a}^{x} \frac{\bar{f}^{\prime}(t)}{(x-t)^{\beta}} d t, \frac{1}{\Gamma(1-\beta)} \int_{a}^{x} \frac{\underline{f}^{\prime}(t)}{(x-t)^{\beta}} d t\right]
$$

when $\mathrm{f}$ is (2)-differentiable, in which $\left(I_{a^{+}}^{\beta} f\right)(x)=\frac{1}{\Gamma(\beta)} \int_{a}^{x} \frac{f(t) d t}{(x-t)^{1-\beta}}$ for $x>a$.

\section{The Fuzzy Laplace Transforms}

Below, we define the fuzzy Laplace transform for a fuzzy-valued function. After that, a derivative theorem is presented to connect the Laplace transform of the fractional derivative and the corresponding fuzzy-valued function. We recall that, firstly, Allahviranloo and Ahmadi [30] suggested the concept of Laplace transforms for fuzzy-valued function as follows:

Definition 9. (see[30]) Let $f(x)$ be a continuous fuzzy-value function; suppose that $f(x) \odot e^{-p x}$ is improper fuzzy Riemann-integrable on $[0, \infty)$; then $\int_{0}^{\infty} f(x) \odot e^{-p x} d x$ is the fuzzy Laplace transforms and can be denoted as:

$$
\boldsymbol{L}\{f(x)\}=\int_{0}^{\infty} f(x) \odot e^{-p x} d x(p>0 \text { and integer })
$$

From Theorem 1, such that $r \in[0,1]$, we conclude:

$$
\int_{0}^{\infty} f(x ; r) \odot e^{-p x} d x=\left[\int_{0}^{\infty} \underline{f}(x ; r) e^{-p x} d x, \int_{0}^{\infty} \bar{f}(x ; r) e^{-p x} d x\right] .
$$

By virtue of the definition of the classical Laplace transform:

$$
\ell\{\underline{f}(x ; r)\}=\int_{0}^{\infty} \underline{f}(x ; r) e^{-p x} d x, \ell\{\bar{f}(x ; r)\}=\int_{0}^{\infty} \bar{f}(x ; r) e^{-p x} d x,
$$

we conclude:

$$
\boldsymbol{L}\{f(x ; r)\}=[\ell\{\underline{f}(x ; r)\}, \ell\{\bar{f}(x ; r)\}] .
$$

Theorem 6. (see [30]) Let $f(x), g(x)$ be continuous-fuzzy-valued functions; suppose that $c_{1}, c_{2}$ are constants, then:

$$
\boldsymbol{L}\left\{\left(c_{1} \odot f(x)\right)+\left(c_{2} \odot g(x)\right)\right\}=c_{1} \odot \boldsymbol{L}\{f(x)\}+c_{2} \odot \boldsymbol{L}\{g(x)\} .
$$

Lemma 1. (see [30]) Let $f(x)$ be a continuous fuzzy-value function on $[0, \infty)$ and $\lambda \in R$, then:

$$
\boldsymbol{L}\{\lambda \odot f(x)\}=\lambda \odot \boldsymbol{L}\{f(x)\} .
$$

Lemma 2. (see [30]) Let $f$ be a continuous fuzzy-value function and $g(x) \geq 0$ a real value function; suppose that $(f(x) \odot g(x)) \odot e^{-p x}$ is improper fuzzy Riemann-integrable on $[0, \infty)$; then, for fixed $r \in[0,1]:$

$$
\int_{0}^{\infty}(f(x ; r) \odot g(x)) \odot e^{-p x} d x=\left[\int_{0}^{\infty} g(x) \underline{f}(x ; r) e^{-p x} d x, \int_{0}^{\infty} g(x) \bar{f}(x ; r) e^{-p x} d x\right] .
$$


Theorem 7. (see [30]) Let $f$ be a continuous fuzzy value function and $\boldsymbol{L}\{f(x)\}=F(p)$; then:

$$
\boldsymbol{L}\left\{e^{a x} \odot f(x)\right\}=F(p-a)
$$

where $e^{a x}$ is a real value function.

\subsection{Proposed Solution}

The fuzzy Laplace transform of Caputo's H-derivative of $f,\left({ }^{C} D_{a^{+}}^{\beta} f\right)(x)$ is crucial in order to obtain the solution of FFDEs. The virtue of $\mathbf{L}\left\{\left({ }^{C} D_{a^{+}}^{\beta} f\right)(x)\right\}$ is related to the fact that it can be written in terms of $\mathbf{L}\{f(x)\}$ :

Theorem 8. Derivative theorem: Suppose that $f$ is a continuous fuzzy-valued function on $[0, \infty)$. Then, we have:

$$
\mathbf{L}\left\{\left({ }^{C} D_{a^{+}}^{\beta} f\right)(x)\right\}=p^{\beta} \odot \mathbf{L}\{f(x)\} \ominus f(0)
$$

if $f$ is ${ }^{C}[(1)-\beta]$-differentiable and:

$$
\mathbf{L}\left\{\left({ }^{C} D_{a^{+}}^{\beta} f\right)(x)\right\}=-f(0) \ominus\left(-p^{\beta} \odot \mathbf{L}\{f(x)\}\right)
$$

if $f$ is ${ }^{C}[(2)-\beta]$-differentiable.

Proof. For arbitrary fixed $r \in[0,1]$, we have:

$$
\left.p^{\beta} \mathbf{L}\{f(x)\} \ominus f(0)=\left[p^{\beta} \ell\{\underline{f}(x ; r)\}-\underline{f}(0 ; r), p^{\beta} \ell\{\bar{f}(x ; r)\}-\bar{f}(0 ; r)\right)\right] .
$$

Since $f$ is ${ }^{C}[(1)-\beta]$,

$$
\begin{aligned}
\left({ }^{C} D_{a^{+}}^{\beta} f\right)(x ; r) & =\left[\left({ }^{C} D_{a^{+}}^{\beta} f\right)(x ; r),\left(\overline{{ }^{C} D_{a^{+}}^{\beta} f}\right)(x ; r)\right] \\
& =\left[\left({ }^{C} D_{a^{+}}^{\beta} f\right)(x ; r),\left({ }^{C} D_{a^{+}}^{\beta} \bar{f}\right)(x ; r)\right] .
\end{aligned}
$$

Hence, we have:

$$
\begin{aligned}
\ell\left\{\left(\overline{{ }^{C} D_{a^{+}}^{\beta} f}\right)(x ; r)\right\} & =\ell\left\{\left({ }^{C} D_{a^{+}}^{\beta} \bar{f}\right)(x ; r)\right\} \\
& =p^{\beta} \ell\{\bar{f}(x ; r)\}-\bar{f}(0 ; r), \\
\ell\left\{\left({ }^{C} D_{a^{+}}^{\beta} f\right)(x ; r)\right\} & =\ell\left\{\left({ }^{C} D_{a^{+}}^{\beta} \underline{f}\right)(x ; r)\right\} \\
& =p^{\beta} \ell\{\underline{f}(x ; r)\}-\underline{f}(0 ; r) .
\end{aligned}
$$

Then, we conclude that:

$$
p^{\beta} \odot \mathbf{L}\{f(x)\} \ominus f(0)=\left[\ell\left\{\left({ }^{C} D_{a^{+}}^{\beta} \underline{f}\right)(x, r)\right\}, \ell\left\{\left({ }^{C} D_{a^{+}}^{\beta} \bar{f}\right)(x ; r)\right\}\right] .
$$

By linearity of $\mathbf{L}$,

$$
p^{\beta} \odot \mathbf{L}\{f(x)\} \ominus f(0)=\mathbf{L}\left\{\left[\left({ }^{C} D_{a^{+}}^{\beta} \underline{f}\right)(x ; r),\left({ }^{C} D_{a^{+}}^{\beta} \bar{f}\right)(x ; r)\right]\right\} .
$$


Using Equation (13), this leads to obtaining:

$$
p^{\beta} \odot \mathbf{L}\{f(x)\} \ominus f(0)=\mathbf{L}\left\{\left({ }^{C} D_{a^{+}}^{\beta} f\right)(x)\right\} .
$$

Now, we assume that $f$ is ${ }^{C}[(2)-\beta]$-differentiable; then for arbitrary fixed $r \in[0,1]$, we have:

$$
-f(0) \ominus\left(-p^{\beta} \mathbf{L}\{f(x)\}\right)=\left[-\bar{f}(0 ; r)+p^{\beta} \ell\{\bar{f}(x ; r)\},-\underline{f}(0, r)+p^{\beta} \ell\{\underline{f}(x, r)\}\right]
$$

and since $f$ is ${ }^{C}[(2)-\beta]$-differentiable, we get:

$$
\begin{aligned}
\left({ }^{C} D_{a^{+}}^{\beta} f\right)(x ; r) & =\left[\left({ }^{C} D_{a^{+}}^{\beta} f\right)(x ; r),\left(\overline{{ }^{C} D_{a^{+}}^{\beta} f}\right)(x ; r)\right] \\
& =\left[\left({ }^{C} D_{a^{+}}^{\beta} \bar{f}\right)(x ; r),\left({ }^{C} D_{a^{+}}^{\beta} f\right)(x ; r)\right] .
\end{aligned}
$$

Therefore, we have:

$$
\begin{aligned}
-f(0) \ominus\left(-p^{\beta} \odot \mathbf{L}\{f(x)\}\right) & =\left[p^{\beta} \ell\{\bar{f}(x ; r)\}-\bar{f}(0 ; r), p^{\beta} \ell\{\underline{f}(x ; r)\}-\underline{f}(0 ; r)\right], \\
& =\mathbf{L}\left\{\left[\left({ }^{C} D_{a^{+}}^{\beta} \bar{f}\right)(x ; r),\left({ }^{C} D_{a^{+}}^{\beta} \underline{f}\right)(x ; r)\right]\right\} .
\end{aligned}
$$

Finally, we can deduce:

$$
-f(0) \ominus\left(-p^{\beta} \odot \mathbf{L}\{f(t)\}\right)=\mathbf{L}\left\{\left({ }^{C} D_{a^{+}}^{\beta} f\right)(x)\right\} .
$$

which finishes the proof.

\section{FFDEs under Caputo's H-Differentiability}

In this section, we investigate the solutions of FFDE under Caputo's H-differentiability with the proposed method. Firstly, we consider the FFDE of order $0<\beta<1$ with the fuzzy initial condition as follows:

$$
\left\{\begin{array}{l}
\left({ }^{C} D_{a^{+}}^{\beta} y\right)(x)=f(x, y(x)) \\
y(a) \in E
\end{array}\right.
$$

The Laplace transform of Equation (17) gave us:

$$
\mathbf{L}\left\{\left({ }^{C} D_{a^{+}}^{\beta} y\right)(x)\right\}=\mathbf{L}\{f(x, y(x)\} .
$$

Thus, using the types Caputo's H-differentiability, we discuss the following cases:

Case I: We consider $y(x)$ a $^{C}[(1)-\beta]$-differentiable function; then Equation (18) is extended based on its lower and upper functions for $0<\beta<1$ as:

$$
\begin{cases}\ell\{\underline{f}(x, y(x) ; r)\}=p^{\beta} \ell\{\underline{y}(x ; r)\}-\underline{y}(0 ; r), & 0 \leq r \leq 1, \\ \ell\{\bar{f}(x, y(x) ; r)\}=p^{\beta} \ell\{\bar{y}(x ; r)\}-\bar{y}(0 ; r), & 0 \leq r \leq 1,\end{cases}
$$

where:

$$
\begin{aligned}
& \underline{f}(x, y(x) ; r)=\min \{f(x, u) \mid u \in[\underline{y}(x ; r), \bar{y}(x ; r)]\}, \\
& \bar{f}(x, y(x) ; r)=\max \{f(x, u) \mid u \in[\underline{y}(x ; r), \bar{y}(x ; r)]\} .
\end{aligned}
$$


In order to find a solution of the linear system (Equation (19)), for simplicity, we make the assumption that:

$$
\ell\{\underline{y}(x ; r)\}=H_{1}(p ; r), \ell\{\bar{y}(x ; r)\}=K_{1}(p ; r),
$$

where $H_{1}(p ; r)$ and $K_{1}(p ; r)$ are solutions of System (19). By using the inverse Laplace transform, $\underline{y}(x ; r)$ and $\bar{y}(x ; r)$ are calculated as follows:

$$
\underline{y}(x ; r)=\ell^{-1}\left\{H_{1}(p ; r)\right\}, \bar{y}(x ; r)=\ell^{-1}\left\{K_{1}(p ; r)\right\},
$$

In this case, we use the symbol $\mathbf{L}^{-1}$ as the inverse of fuzzy Laplace transform, and it is equivalent to:

$$
\left(\ell^{-1}\left\{H_{1}(p ; r)\right\}, \ell^{-1}\left\{K_{1}(p ; r)\right\}\right) .
$$

Case II: Let us consider $y(x)$ as ${ }^{C}[(2)-\beta]$-differentiable; then for $0<\beta<1$, Equation (18) can be written as:

$$
\left\{\begin{array}{l}
\ell\{\underline{f}(x, y(x) ; r)\}=p^{\beta} \ell\{\underline{y}(x ; r)\}-\underline{y}(0 ; r), \\
\ell\{\bar{f}(x, y(x) ; r)\}=p^{\beta} \ell\{\bar{y}(x ; r)\}-\bar{y}(0 ; r),
\end{array}\right.
$$

where:

$$
\begin{aligned}
& \underline{f}(x, y(x) ; r)=\min \{f(x, u) \mid u \in[\underline{y}(x ; r), \bar{y}(x ; r)]\}, \\
& \bar{f}(x, y(x) ; r)=\max \{f(x, u) \mid u \in[\underline{y}(x ; r), \bar{y}(x ; r)]\} .
\end{aligned}
$$

Similar to Case I, in order to solve the linear system (Equation (23)), we simplify:

$$
\begin{aligned}
& \ell\{\underline{y}(x ; r)\}=H_{2}(p ; r), \\
& \ell\{\bar{y}(x ; r)\}=K_{2}(p ; r),
\end{aligned}
$$

where $\mathrm{H}_{2}(p ; r)$ and $K_{2}(p ; r)$ are solutions of the system (Equation (23)). By using the inverse Laplace transform, $\underline{y}(x ; r)$ and $\bar{y}(x ; r)$ are computed as follows:

$$
\begin{aligned}
& \underline{y}(x ; r)=\ell^{-1}\left\{H_{2}(p ; r)\right\}, \\
& \bar{y}(x ; r)=\ell^{-1}\left\{K_{2}(p ; r)\right\},
\end{aligned}
$$

and this is equivalent to:

$$
\left(\ell^{-1}\left\{H_{2}(p ; r)\right\}, \ell^{-1}\left\{K_{2}(p ; r)\right\}\right) .
$$

\section{Application}

In this section, we will solve some real-world examples (to obtain the solutions of FFDE) under the definition of Caputo differentiability for a fuzzy-valued function about fractional order $0<\beta<1$. Firstly, we solve a homogeneous FFDE (Example 1). Then, in a general case, we solve an example that includes the free parameter $\lambda$ (Example 2), which has appeared in the fuzzy literature several times under Caputo's H-differentiability [32]. Finally, the fuzzy Basset problem (Example 3) is determined to show the ability of the proposed method.

This paper is the first attempt that provides analytical solutions for the FFDEs under the Caputo differentiability. Hence, there are no other reported analytical methods for such problems to compare in the fuzzy Laplace method. 
Example 1. We analyze the following FFDE:

$$
\begin{cases}\left({ }^{C} D_{0^{+}}^{\beta} y\right)(x)=0, & 0<\beta<1 \\ y(0 ; r)=\left[\underline{y}_{0}(r), \bar{y}_{0}(r)\right], & 0 \leq r \leq 1 .\end{cases}
$$

With the help of the Laplace transforms on Equation (26), we obtain:

$$
\mathbf{L}\left\{\left({ }^{C} D_{0^{+}}^{\beta} y\right)(x)\right\}=\mathbf{L}\{0\} .
$$

The inverse of the Laplace on Equation (27) gave us:

$$
\begin{aligned}
& \underline{y}(x ; r)=\ell^{-1}\left\{\frac{\underline{y}(0 ; r)}{p^{\beta}}\right\}, 0 \leq r \leq 1,0<\beta<1, \\
& \bar{y}(x ; r)=\ell^{-1}\left\{\frac{\bar{y}(0 ; r)}{p^{\beta}}\right\}, 0 \leq r \leq 1,0<\beta<1
\end{aligned}
$$

which led to deriving lower and upper functions of the solution for $0<\beta<1$ as follows:

$$
\underline{y}(x ; r)=\underline{y}_{0}(r) x^{\beta-1}, \bar{y}(x ; r)=\bar{y}_{0}(r) x^{\beta-1}, 0 \leq r \leq 1 .
$$

Example 2. In the following, we analyze the following FFDE,

$$
\begin{cases}\left({ }^{C} D_{0^{+}}^{\beta} y\right)(x)=\lambda \odot y(x), & 0<\beta<1 \\ y(0 ; r)=\left[\underline{y}_{0}(r), \bar{y}_{0}(r)\right], & 0 \leq r \leq 1 .\end{cases}
$$

such that $y(x)$ is the number of radionuclides present in a given radioactivity, and $\lambda$ is a decay constant. We investigate the solution in this case for $\lambda \in R$.

Case I: We make the assumption that $\lambda \in R^{+}=(0,+\infty)$, and after that, we use the Laplace transform and finally conclude:

$$
\mathbf{L}\left\{\left({ }^{C} D_{a^{+}}^{\beta} y\right)(x)\right\}=\mathbf{L}\{\lambda \odot y(x)\} .
$$

Using ${ }^{C}[(1)-\beta]$-differentiability, we get:

$$
\left\{\begin{array}{l}
\lambda \ell\{\underline{y}(x ; r)\}=p^{\beta} \ell\{\underline{y}(x ; r)\}-\underline{y}(0 ; r) \\
\lambda \ell\{\bar{y}(x ; r)\}=p^{\beta} \ell\{\bar{y}(x ; r)\}-\bar{y}(0 ; r) .
\end{array}\right.
$$

After some manipulations, we get the following:

$$
\left\{\begin{aligned}
\left(p^{\beta}-\lambda\right) \ell\{\underline{y}(x ; r)\} & =\underline{y}(0 ; r), \\
\left(p^{\beta}-\lambda\right) \ell\{\bar{y}(x ; r)\} & =\bar{y}(0 ; r) .
\end{aligned}\right.
$$

The inverse of the fuzzy Laplace transforms on Equation (33) leads us to:

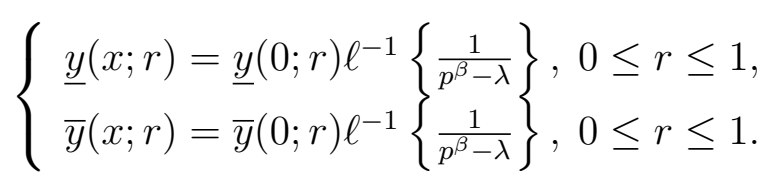


Finally, the solution of FFDE is determined as follows:

$$
\begin{cases}y(x ; r)=\underline{y}_{0}(r) E_{\beta, 1}\left[\lambda x^{\beta}\right], & 0 \leq r \leq 1 \\ \bar{y}(x ; r)=\bar{y}_{0}(r) E_{\beta, 1}\left[\lambda x^{\beta}\right], & 0 \leq r \leq 1\end{cases}
$$

where $E_{\beta, 1}$ is the Mittag-Leffler function (see [11]).

Case II: Suppose that $\lambda \in R^{-}=(-\infty, 0)$; then, using ${ }^{C}[(2)-\beta]$-differentiability and Theorem 8 , the obtained solution will be similar to Equation (34). For a special case, let us consider $\beta=0.5, \lambda=1$ and $y(0 ; r)=[1+r, 3-r]$; then, the solution for Case I is derived as follows:

$$
y(x ; r)=[1+r, 3-r] \odot\left(\frac{1}{\sqrt{\pi x}}+e^{x} \operatorname{erfc}(-\sqrt{x})\right),
$$

and the solution for Case II with $\lambda=-1$ is obtained as follows:

$$
y(x ; r)=[1+r, 3-r] \odot\left(\frac{1}{\sqrt{\pi x}}-e^{x} \operatorname{erfc}(\sqrt{x})\right)
$$

where $\operatorname{erfc}(x)=\frac{2}{\sqrt{\pi}} \int_{x}^{\infty} e^{-t^{2}} d t$

Example 3. The Basset problem: The dynamics of a sphere immersed in an incompressible viscous fluid is a classical problem with huge applications in material sciences, as well as in the study of geophysical flows. A particularly important problem is the study of a sphere subjected to gravity, which was first presented by Basset in 1888 [51] and followed in 1910 by [52], who then introduced a special hydraulic force, known as "Basset's force".

Basset's force was studied by Mainardi $[53,54]$ in terms of a fractional derivative of order $\frac{1}{2}$ of the velocity of the particle relative to the fluid and is recognized as the generalized Basset's force. It is based on the fractional Caputo derivative $(0<\beta<1)$ and on a generalized model of [52]. That is, when the fluid is at rest and the particle moves vertically under the effect of gravity, with a certain initial velocity $y_{0}$ :

$$
y^{\prime}(x)+2 \odot\left({ }^{C} D_{0^{+}}^{\beta} y\right)(x)+y(x)=g(x)
$$

Now, in order to discover this in a real concept, we use the fuzzy initial value $y_{0}$, the fuzzy-valued function $g(x)$ and the concept of Caputo's H-differentiability for the fractional derivative of $y(x)$, $\left({ }^{C} D_{0^{+}}^{\beta} y\right)(x)$ and the generalized H-differentiability [32] for the first order derivative of $y(x), y^{\prime}(x)$. Let us consider the fuzzy version of the dynamics of a sphere immersed in an incompressible viscous fluid (Basset's problem) as follows:

$$
\left\{\begin{aligned}
y^{\prime}(x)+2 \odot\left({ }^{C} D_{0^{+}}^{\beta} y\right)(x)+y(x)=g(x), & 0<\beta<1 \\
y(0 ; r)=0, & 0 \leq r \leq 1
\end{aligned}\right.
$$

where $g$ is an arbitrary fuzzy-valued function, which can possess the fuzzy Laplace transforms.

In order to solve this example under ${ }^{C}[(1)-\beta]$-differentiability, we apply the fuzzy Laplace transform to both sides of Equation (37), which leads to:

$$
\mathbf{L}\left\{y^{\prime}(x)\right\}+2 \odot \mathbf{L}\left\{\left(D_{0^{+}}^{\beta} y\right)(x)\right\}+\mathbf{L}\{y(x)\}=\mathbf{L}\{g(x)\}
$$


Simply using ${ }^{C}[(1)-\beta]$-differentiability, we get:

$$
\begin{aligned}
p \odot \mathbf{L}\{y(x)\} \quad & y(0)+2 p^{\beta} \odot \mathbf{L}\{y(x)\} \\
& \ominus \quad y(0)+\mathbf{L}\{y(x)\}=\mathbf{L}\{g(x)\} .
\end{aligned}
$$

Then, after simple computations, we get:

$$
\mathbf{L}\{y(x)\}=\frac{\mathbf{L}\{g(x)\}+2 \odot y(0)}{p+2 p^{\beta}+1} .
$$

Applying the inverse of fuzzy Laplace transforms, we have:

$$
y(x)=\mathbf{L}^{-1}\left\{\frac{\mathbf{L}\{g(x)\}+2 \odot y(0)}{p+2 p^{\beta}+1}\right\} .
$$

For a special case, let us consider $\beta=\frac{1}{2}$ and $g(x ; r)=[-2+2 r, 2-2 r]$. Then, our solution is:

$$
y(x ; r)=[-2+2 r, 2-2 r] \odot \mathbf{L}^{-1}\left\{\frac{1}{p\left(p^{\frac{1}{2}}+1\right)^{2}}\right\}
$$

or, equivalently:

$$
y(x ; r)=[-2+2 r, 2-2 r] \odot\left(1+e^{x}(2 x-1) \operatorname{erfc}\left(x^{\frac{1}{2}}\right)-2\left(\frac{x}{\pi}\right)^{\frac{1}{2}}\right) .
$$

\section{Conclusion and Future Works}

In this paper, we investigated an analytical solution of the FFDEs (Equation (17)), under Caputo's H-differentiability. To this end, we revisited Caputo's derivatives, and proposed novel fuzzy Laplace transforms and their inverses, with an analytical method to tackle the deficiencies in the state-of-the-art methods (Equation (22) under ${ }^{C}[(1)-\beta]$ Caputo H-differentiability, Equations (24) and (25) under ${ }^{C}[(2)-\beta]$ Caputo H-differentiability). Experimental results using some real-world problems (nuclear decay Equation (30) and Basset problem (38)) illustrated the effectiveness and applicability of the proposed method.

\section{Acknowledgment}

The authors would like to thank Chee Seng Chan, Centre of Image and Signal Processing, Faculty of Computer Science \& Information Technology, University of Malaya, Kuala Lumpur, Malaysia, for his valuable remakrs which improved the paper.

\section{Author Contributions}

Soheil Salahshour and Ali Ahmadian wrote the first draft. Noraza Senu and Praveen Agarwal corrected and improved it, and Dumitru Baleanu prepared the final version. All authors have read and approved the final manuscript. 


\section{Conflicts of Interest}

The authors declare no conflict of interest.

\section{References}

1. Arara, A.; Benchohra, M.; Hamidi, N.; Nieto, J. Fractional order differential equations on an unbounded domain. Nonlinear Anal. Theory Methods Appl. 2010, 72, 580-586.

2. Bagley, R.L. On the fractional order initial value problem and its engineering applications. Fract. Calc. Appl. 1990, 12-20.

3. Beyer, H.; Kempfle, S. Definition of physically consistent damping laws with fractional derivatives. Zeitschrift für Angewandte Mathematik und Mechanik 1995, 75, 623-635.

4. Diethelm, K.; Ford, N. Analysis of fractional differential equations. J. Math. Anal. App. 2002, 265, 229-248.

5. Mainardi, F. Fractional relaxation-oscillation and fractional diffusion-wave phenomena. Chaos Solitons Fractals 1996, 7, 1461-1477.

6. Ingo, C.; Magin, R.L.; Parrish, T.B. New Insights into the Fractional Order Diffusion Equation Using Entropy and Kurtosis. Entropy 2014, 16, 5838-5852.

7. Baleanu, D.; Güvenc, Z.; Tenreiro Machado, J. New Trends in Nanotechnology and Fractional Calculus Applications; Springer-Verlag: Berlin, Germany, 2010.

8. Dorčák, L.; Valsa, J.; Gonzalez, E.; Terpák, J.; Petráš, I.; Pivka, L. Analogue Realization of Fractional-Order Dynamical Systems . Entropy 2013, 15, 4199-4214.

9. Lakshmikantham, V.; Leela, S.; Devi, J. Theory of Fractional Dynamic Systems; Cambridge Scientific Publishers: Cambridge, UK, 2009.

10. Podlubny, I. Fractional Differential Equations: An Introduction to Fractional Derivatives, Fractional Differential Equations, to Methods of Their Solution and Some of Their Applications; Elsevier Science: Amsterdam, The Netherlands, 1998.

11. Kilbas, A.A.; Srivastava, H.M.; Trujillo, J.J. Theory and Applications of Fractional Differential Equations; Elsevier Science: Amsterdam, The Netherlands, 2006.

12. Baleanu, D.; Diethelm, K.; Scalas, E.; Trujillo, J. Fractional Calculus: Models and Numerical Methods; World Scientific: Singapore, Singapore, 2012.

13. Khan, Y.; Wu, Q.; Faraz, N.; Yildirim, A.; Madani, M. A new fractional analytical approach via a modified Riemann-Liouville derivative. Appl. Math. Lett. 2012, 25, 1340-1346.

14. Lakshmikantham, V.; Vatsala, A.S. General uniqueness and monotone iterative technique for fractional differential equations. Appl. Math. Lett. 2012, 25, 1340-1346.

15. El-Ajou, A.; Abu Arqub, O.; Al Zhour, Z.; Momani, S. New Results on Fractional Power Series: Theories and Applications. Fuzzy Sets Syst. 2013, 15, 5305-5323.

16. Momani, S.; Aslam Noor, M. Numerical methods for fourth-order fractional integro-differential equations. Appl. Math. Comput. 2006, 182, 754-760.

17. Bhrawy, A.; Alofi, A.; Ezz-Eldien, S. A quadrature tau method for fractional differential equations with variable coefficients. Appl. Math. Lett. 2011, 24, 2146-2152. 
18. Bhrawy, A.H.; Zaky, M.A. A method based on the Jacobi tau approximation for solving multi-term time-space fractional partial differential equations. J. Comput. Phys. 2015, 281, 876-895.

19. Bhrawy, A.H.; Doha, E.H.; Baleanu, D.; Ezz-Eldien, S.S. A spectral tau algorithm based on Jacobi operational matrix for numerical solution of time fractional diffusion-wave equations. J. Comput. Phys. 2015, in press.

20. Bhrawy, A.H.; Zaky, M.A.; Baleanu, D. New Numerical Approximations for Space-Time Fractional Burgers' Equations via a Legendre Spectral-Collocation Method. Rom. Rep. Phys. 2015, 67, In press.

21. Bhrawy, A.H.; Tharwat, M.M.; Yildirim, A. A new formula for fractional integrals of Chebyshev polynomials: Application for solving multi-term fractional differential equations. Appl. Math. Model. 2013, 37, 4245-4252.

22. Zhang, L.; Ahmad, B.; Wang, G. The existence of an extremal solution to a nonlinear system with the right-handed Riemann-Liouville fractional derivative. Appl. Math. Lett. 2014, 31,1-6.

23. Lakshmikantham, V.; Vatsala, A. Basic theory of fractional differential equations. Nonlinear Anal. Theory Methods Appl. 2008, 69, 2677-2682.

24. Felice, D.; Cafaro, C.; Mancini, S. Information Geometric Complexity of a Trivariate Gaussian Statistical Model. Entropy 2014, 16, 2944-2958.

25. Sun, J.; Cafaro, C.; Bollt, E.M. Identifying the Coupling Structure in Complex Systems through the Optimal Causation Entropy Principle. Entropy 2014, 16, 3416-3433.

26. Agarwal, R.; Lakshmikantham, V.; Nieto, J. On the concept of solution for fractional differential equations with uncertainty. Nonlinear Anal. Theory Methods Appl. 2000, 72, 2859-2862.

27. Allahviranloo, T.; Salahshour, S.; Abbasbandy, S. Explicit solutions of fractional differential equations with uncertainty. Soft Comput. 2012, 16, 297-302.

28. Mazandarani, M.; Vahidian Kamyad, A. Modified fractional Euler method for solving Fuzzy Fractional Initial Value Problem. Commun. Nonlinear Sci. Numer. Simulat. 2013, 18, 12-21.

29. Salahshour, S.; Allahviranloo, T.; Abbasbandy, S. Solving fuzzy fractional differential equations by fuzzy Laplace transforms. Commun. Nonlinear Sci. Numer. Simul. 2012, 17, 1372-1381.

30. Allahviranloo, T.; Ahmadi, M. Fuzzy laplace transforms. Soft Comput. 2010, 14, 235-243.

31. Mazandarani, M.; Najariyan, M. Type-2 fuzzy fractional derivatives. Commun. Nonlinear Sci. Numer. Simul. 2014, 19, 2354-2372.

32. Bede, B.; Gal, S. Generalizations of the differentiability of fuzzy-number-valued functions with applications to fuzzy differential equations. Fuzzy Sets Syst. 2005, 151, 581-599.

33. Bede, B.; Rudas, I.; Bencsik, A. First order linear fuzzy differential equations under generalized differentiability. Inf. Sci. 2007, 177, 1648-1662.

34. Ahmadian, A.; Suleiman, M.; Salahshour, S.; Baleanu, D. A Jacobi operational matrix for solving a fuzzy linear fractional differential equation. Adv. Diff. Equ. 2013, 104, doi:10.1186/1687-1847-2013-104.

35. Ahmadian, A.; Suleiman, M.; Salahshour, S. An operational matrix based on Legendre polynomials for solving fuzzy fractional-order differential equations. Abstr. Appl. Anal. 2013, 2013, doi:10.1155/2013/505903. 
36. Balooch Shahriyar, M.R.; Ismail, F.; Aghabeigi, S.; Ahmadian, A.; Salahshour, S. An Eigenvalue-Eigenvector Method for Solving a System of Fractional Differential Equations with Uncertainty. Math. Probl. Eng. 2013, 2013, doi:10.1155/2013/579761.

37. Salahshour, S.; Allahviranloo, T.; Abbasbandy, S.; Baleanu, D. Existence and uniqueness results for fractional differential equations with uncertainty. $A d v$. Diff. Equ. 2012, 112, doi:10.1186/1687-1847-2012-112.

38. Allahviranloo, T.; Abbasbandy, S.; Balooch Shahryari, M.R.; Salahshour, S.; Baleanu, D. On Solutions of Linear Fractional Differential Equations with Uncertainty. Abstr. Appl. Anal. 2013, 2013, doi:10.1155/2013/178378.

39. Diamond, P. Theory and applications of fuzzy Volterra integral equations. IEEE Trans. Fuzzy Syst. 2002, 157, 97-102.

40. Perfilieva, I. Cauchy problem with fuzzy initial condition and its approximate solution with the help of fuzzy transform. In Proceedings of IEEE International Conference on Fuzzy Systems, 2008 (IEEE World Congress on Computational Intelligence), Hong Kong, China, 1-6 June 2008; pp. 2285-2290.

41. Jafarian, A.; Golmankhaneh, A.R.; Baleanu, D. On Fuzzy Fractional Laplace Transformation. Inf. Control 2014, 2014, doi:10.1155/2014/295432.

42. Diamond, P.; Kloeden, P. Metric Spaces of Fuzzy Sets: Theory and Applications; World Scientific: Singapore, Singapore, 1994.

43. Zimmermann, H. Fuzzy Set Theory And Its Applications; Springer-Verlag: Berlin, Germany, 2001.

44. Friedman, M.; Ma, M.; Kandel, A. Numerical solutions of fuzzy differential and integral equations. Fuzzy Sets Syst. 1999, 106, 35-48.

45. Puri, M.; Ralescu, D. Fuzzy random variables. J. Math. Anal. Appl. 1986, 114, 409-422.

46. $\mathrm{Wu}, \mathrm{H}-\mathrm{C}$. The improper fuzzy Riemann integral and its numerical integration. Inf. Sci. 1998, 111, 109-137.

47. Chalco-Cano, Y.; Román-Flores, H. On new solutions of fuzzy differential equations. Chaos Solitons Fractals 2008, 38, 112-119.

48. Alikhani, R.; Bahrami, F. Global solutions for nonlinear fuzzy fractional integral and integrodifferential equations. Commun. Nonlinear Sci. Numer. Simul. 2013, 18, 2007-2017.

49. Salahshour, S.; Abbasbandy, S. A comment on "Global solutions for nonlinear fuzzy fractional integral and integrodifferential equations”. Commun. Nonlinear Sci. Numer. Simul. 2014, $19,1256-1258$.

50. Lupulescu, V. Fractional calculus for interval-valued functions. Fuzzy Sets Syst. 2014, doi:10.1016/j.fss.2014.04.005.

51. Basset, A. A Treatise on Hydrodynamics: With Numerous Examples; Deighton, Bell and Co.: Cambridge, UK, 1888.

52. Basset, A. The Descent of a Sphere in a Viscous Liquid. Nature 1910, 83, 521.

53. Mainardi, F. Fractional relaxation-oscillation and fractional diffusion-wave phenomena. Chaos Solitons Fractals 1996, 7, 1461-1477. 
54. Mainardi, F.; Pironi, P.; Tampieri, F. On a generalization of the Basset problem via Fractional Calculus. In Proceedings of the 15th Canadian Congress of Applied Mechanics, CANCAM'95, University of Victoria, Victoria, Canada, 28 May-2 June 1995; pp. 836-837.

(c) 2015 by the authors; licensee MDPI, Basel, Switzerland. This article is an open access article distributed under the terms and conditions of the Creative Commons Attribution license (http://creativecommons.org/licenses/by/4.0/). 